\title{
The relationship between total atrial conduction time and left atrial global strain in patients with psoriasis vulgaris
}

Hakan Duman ${ }^{1}$, Nursel Dilek², Selami Demirelli ${ }^{3}$, Sinan Inci ${ }^{4}$, Handan Duman ${ }^{5}$, Mustafa Çetin ${ }^{1}$, Murtaza Emre Durakoğlugil ${ }^{1}$

\author{
${ }^{1}$ Department of Cardiology, Recep Tayyip Erdoğan University, Rize, Turkey \\ ${ }^{2}$ Department of Dermatology, Rize University Medical Faculty, Rize, Turkey \\ ${ }^{3}$ Department of Cardiology, Erzurum Education and Research Hospital, Erzurum, \\ Turkey \\ ${ }^{4}$ Department of Cardiology, Aksaray State Hospital, Aksaray, Turkey \\ ${ }^{5}$ Department of Family Medicine, Recep Tayyip Erdoğan University, Rize, Turkey
}

Submitted: 27 December 2016

Accepted: 30 March 2017

Arch Med Sci 2019; 15 (4): 865-871

DOI: https://doi.org/10.5114/aoms.2019.82678

Copyright $\odot 2019$ Termedia \& Banach

\section{Abstract}

Introduction: Psoriasis vulgaris is a chronic, multisystem disease that results in the development of atrial fibrillation (AF) over time. In this study, our goal was to assess predictors of AF in patients with psoriasis, including total atrial conduction time (TACT) and left atrial global longitudinal strain (LAGLS).

Material and methods: A total of 80 individuals, including 40 psoriasis patients and 40 healthy controls, were enrolled in the study. A physical examination was performed, biochemical parameters were studied, and Holter electrocardiography was carried out. Conventional echocardiography, atrial tissue Doppler, and speckle tracking echocardiography were recorded.

Results: No significant difference was observed between psoriasis patients and healthy controls with regard to age, and the average duration of psoriasis was 5.7 years. High-sensitivity $C$ reactive protein levels were higher in the patient group compared to the control group (respectively, group 1: $1 \pm 0.8$; group 2: $0.6 \pm 0.3, p<0.05$ ). Atrial arrhythmia was not detected in the Holter ECG monitoring. A significant moderate negative correlation between TACT and LAGLS $(r=-0.57, p<0.05)$ was observed, and there was a significant moderate positive correlation between the duration of disease and TACT $(r=0.52, p<0.05)$.

Conclusions: In the current study, we determined that LAGLS decreased, TACT was prolonged, and P-wave dispersion increased in patients with psoriasis. The current results may improve predictions of AF risk in psoriasis patients in clinical practice.

Key words: psoriasis vulgaris, left atrium, global strain.
Corresponding author:

Hakan Duman

Department

of Cardiology

Recep Tayyip

Erdoğan University

53100 Rize, Turkey

Phone: +90 4642236126

E-mail: drhakanduman@

hotmail.com

\section{Introduction}

Psoriasis epidermal hyperproliferation is a chronic, multi-systemic inflammatory disease characterised by abnormal keratinocyte differentiation, T-lymphocyte infiltration, and increased cytokine expression [1]. It is present on the skin in 6-8\% of patients arriving at the Dermatology Clinic [2]. The etiology of this disease is unknown; however, various factors such 
as genetics, trauma, infections, drugs, and smoking are associated with the aetiopathogenesis of the disease. Chronic inflammation and oxidative stress cause an increase in the prevalence of cardiovascular disease [3]. Atherosclerosis, hypertension, and valvular involvement are frequently encountered in patients with psoriasis. However, a recent study by Ahlehoff et al. determined that psoriasis was related to an increased risk of atrial fibrillation (AF) [4]. Other studies have also reported that secondary amyloid deposition was present in the conduction system, and correspondingly, atrial conduction disorders were frequently observed [5, 6]. The AF is the most common arrhythmia, and it is associated with an increased risk of stroke, heart failure, coronary artery disease, and cardiovascular mortality. Total atrial conduction time (TACT) is detected using echocardiography, and is prolonged in cases of discontinuous and heterogeneous propagation of sinus impulses [7]. Weijs et al. determined that prolonged TACT and AF were related [8]. Left atrial global longitudinal strain (LAGLS), which is detected using two-dimensional speckle tracking echocardiography (2D-STE), is a new tool currently under investigation for the prediction of AF. The determination of the relationship between TACT and LAGLS will contribute to the ability to predict $A F$, which is an arrhythmia with a high rate of morbidity and mortality in psoriasis patients, and is frequently seen in the clinic.

There are currently no studies exploring the role of LAGLS and TACT in the induction of subclinical electrophysiological changes in psoriasis patients; therefore, the goal of the current study was to investigate the relationship between TACT and LAGLS in patients with psoriasis.

\section{Material and methods}

This study was performed in accordance with the Helsinki Declaration after consent was obtained from the hospital Ethics Committee. All patients were informed prior to beginning the study, and their approval was granted.

\section{Study population}

Forty patients who were over the age of 18 years, had been diagnosed with psoriasis vulgaris for a minimum of 3 years via biopsy, and admitted to the Dermatology Department, Faculty of Medicine, Recep Tayyip Erdoğan University, Rize, Turkey (group 1), were selected as study participants. Additionally, 40 age- and sex-matched healthy individuals were included in this study as the control group (group 2). Patients and control subjects with a history of past or concurrent diseases such as hypertension $(\mathrm{HT})$, diabetes mellitus, coronary artery disease (CAD), lung diseases and/or pul- monary HT, valvular heart diseases, liver or kidney diseases, collagen vascular diseases, rhythms other than sinus, any cardiovascular drug use, abnormal thyroid function, or serum electrolyte values were excluded from the study. Also, obese subjects $\left(B M I \geq 30 \mathrm{~kg} / \mathrm{m}^{2}\right)$ were excluded from the study. All subjects gave informed consent, and the local Ethics Committee approved the study. Patients with coexisting psoriatic arthritis (diagnosed by a rheumatologist) were excluded due to the possible adverse effects on heart rhythm. The age, gender, age of onset, duration of the disease, and drug history of each patient were recorded. Height, weight, body mass index (BMI), and waist circumference were also assessed in all patients and biochemical variables such as fasting glucose levels and a lipid panel were also analysed and Holter electrocardiography (ECG) was recorded.

\section{Study design}

A total of 80 patients, of whom 40 were psoriasis patients and the remaining 40 healthy controls, were included in the study. The group of psoriasis patients was named group 1 , and the control group was named group 2 .

\section{Standard echocardiographic evaluation}

The echocardiographic assessment was performed on patients during clinical remission in the routine follow-up period. Also, none of the patients used any drugs that affected the heart during the echocardiographic assessment. Transthoracic echocardiographic assessments were carried out using the S5-1 probe $(5-1 \mathrm{MHz})$ transducer with a Philips iE33 Medical ultrasound Systems device. The patients were assessed in a left lateral decubitus position after a 5-minute rest period. Firstly, the pericardium, valve morphology, and wall motions were assessed using $M$ mode and two-dimensional echocardiography. The LV ejection fraction (LVEF) and left atrium diameter (LA) were calculated using the parasternal long axis. In pulse wave (PW) Doppler examination of LV filling using an apical four-chamber view, the Doppler sample volume in parallel to the LV long axis and at the mitral annulus were measured and averaged. During the assessment, the early diastolic flow velocity $(E)$, the late diastolic flow velocity (A), E/Em, the deceleration time (DT), and the isovolumetric relaxation time (IVRT) were recorded. In the apical four-chamber view, a 5-mm-width PWD sample volume was performed by placement on the point of intersection of the posterior wall and the mitral annulus. By ensuring that the sample volume was parallel with the wall axis, peak early (Em) diastolic flow velocity was recorded. LA volumes were determined using the biplane 'area-length' method 
from apical four- and two-chamber images, and their average was calculated. The LA maximum volume (LAVmax) was measured when the mitral valve was completely opened, and LA minimum volume (LAVmin) was measured when the mitral valve was completely closed, and the following parameters were calculated using these measurements: LAVreservoir (ml): LAVmax-LAVmin. All measurements were repeated during three consecutive heartbeats and averaged. The American Society of Echocardiography's standards were used as a base for all the measurements [9].

\section{D speckle tracking echocardiography}

The apical four-chamber and two-chamber grey scale views were stored digitally. The records were then processed by TDI data and analysed off-line using dedicated software (QLAB, Philips). The global strain and the strain rate were calculated by averaging the values measured in 15 atrial segments. The frame rate was $50-80 \mathrm{frame} / \mathrm{s}$. The global peak systolic LA myocardium strain during the left ventricular systole (LAGLSs), global peak systolic positive LA myocardial strain rate during left ventricular systole (LAGLSRs), global peak negative LA myocardial strain rate during early ventricular diastole (LAGLSRe), and global peak negative LA myocardial strain rate (LAGLSRa) during late ventricular diastole were obtained (Figure 1).

\section{Atrial tissue Doppler imaging}

Total atrial conduction time - the time from initiation of the electrocardiographic P-wave (lead II) to the local lateral left atrial activation time [10] - was determined using transthoracic echocardiography. This was measured by placing the sample volume in the left atrial basal portion and then measuring the time between initiation of the electrocardiographic P-wave and the peak velocity, using tissue velocity imaging (Figure 2).

\section{Evaluation of patients' disease activity}

The diagnosis of psoriasis vulgaris was performed by a dermatologist and/or was based on a description of characteristic lesions. The clinical severity was determined according to the Psoriasis Area and Severity Index (PASI) [11]. The PASI assesses four body regions: the head, trunk, upper extremities, and lower extremities. For each region, the surface area involved is graded from 0 to 6 , and each of the three variables (erythema, thickness, and scaling of the plaques) is graded from 0 to 4 . The scores from each of the regions were added to determine a PASI score ranging from 0 to 72 . The Psoriasis Severity Index (PSI) was also used to evaluate the clinical signs (erythema, thickness, and scaling) on a scale of 0 (absent) to 3 (severe)
[12]. The affected body surface area (BSA) was also evaluated. The Nail Psoriasis Severity Index (NAPSI) - a simple numerical tool for the evaluation of nail psoriasis - was used to quantify the degree of nail changes [13]. The NAPSI was assessed separately for each fingernail and toenail. This scale is used to evaluate the severity of nail-bed psoriasis and nail-matrix psoriasis. Each nail was evaluated for the presence or absence of nail-matrix disease (pitting, leukonychia, red spots in the lunula, nailplate crumbling) and nail-bed disease (oil drop/ salmon patch discolouration, onycholysis, nail-bed hyperkeratosis, and splinter haemorrhage). The sum of the scores for all of the nails is the patient's NAPSI. Nail involvement was considered for the patients with a NAPSI score $\geq 1$.

\section{Twelve-lead electrocardiogram and P-wave duration analysis}

A 12-lead ECG of all patients was recorded in the supine position (Montara Instrument EU 250 Electrocardiograph, Milwaukee, WI, USA). ECG recordings were obtained at a paper speed of

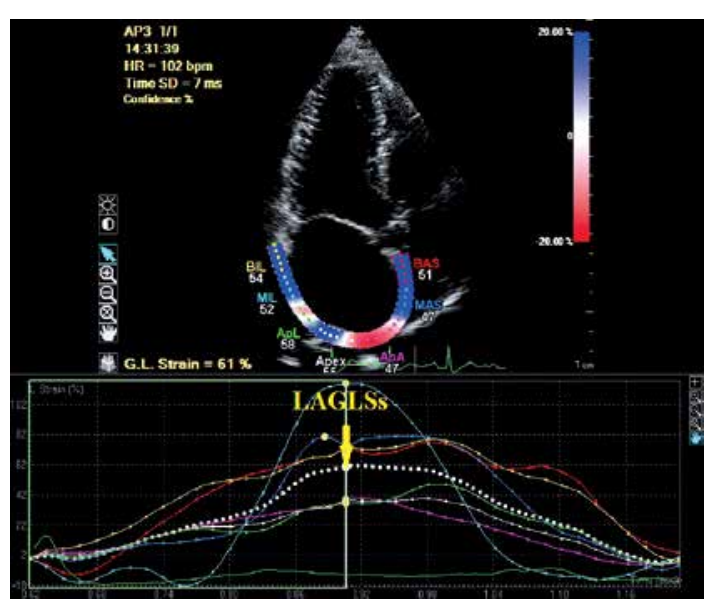

Figure 1. Measurement of left atrial systolic global longitudinal strain (LAGLSs)

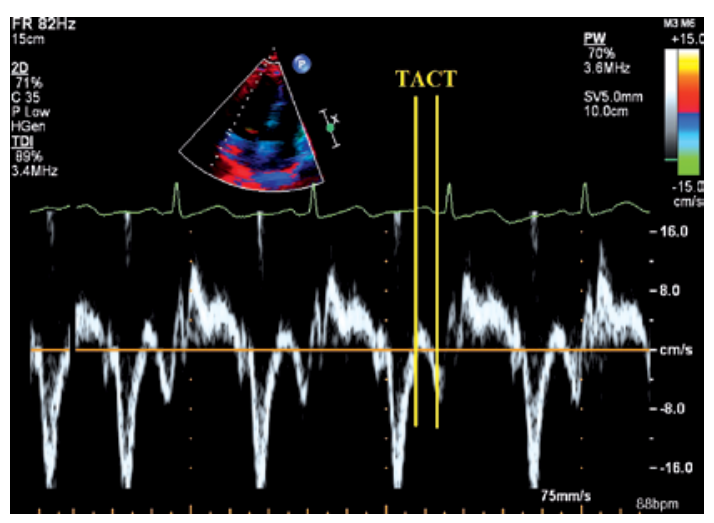

Figure 2. Measurement of the time between the initiation of the electrocardiographic P-wave and the peak velocity using tissue velocity imaging TACT - total atrial conduction time. 
$50 \mathrm{~mm} / \mathrm{s}$ and $10 \mathrm{~mm} / \mathrm{mV}$ amplitude. The beginning of the P-wave was defined as the point at which the first atrial deflection crossed the isoelectric line, and the end of the P-wave was defined as the point at which the atrial deflection returned to the isoelectric line. The P-wave durations (Pmax, Pmin) were calculated in all 12 ECG leads. The difference between Pmax and Pmin was defined as P-wave dispersion (PWD) [14]. Two ECG readers, who were blinded to the study, evaluated the ECGs. Initially, the measurements were performed manually with the help of callipers and a magnifying glass to define the electrocardiographic deflection.

\section{Reproducibility}

Bland-Altman analyses were performed in our laboratory to evaluate the inter- and intra-observer reproducibility of the total atrial conduction time measurements, which demonstrated minimal biases and tight limits of agreement $(1.8+10$ and $1.7+10 \mathrm{~ms}$, respectively). The intra- and inter-observer reproducibility for the left atrium peak longitudinal strain was also evaluated. For the intra-observer assessment, images from 20 randomly selected patients were reanalysed after 1 week. The Bland-Altman analysis and the intraclass correlation coefficient (ICC) indicated good interand intra-observer agreement: the inter-observer agreement for the left atrium global longitudinal peak strain had an average difference of $2.0(-1.9$, 4.5) and the ICC was 0.89, while the intra-observer agreement determined that the average difference was $1.5(-2.1,3.2)$ and the ICC was 0.93 .

\section{Statistical analysis}

The continuous variables are presented as means \pm standard deviation, and the categorical variables as percentages. The Kolmogorov-Smirnov test was used to verify the normality of the distribution of the continuous variables. The statistical analysis of the clinical data between the two groups consisted of unpaired $t$-tests for parametric data and the Mann-Whitney $U$ test analysis for nonparametric data. The correlations were assessed by means of Pearson and Spearman correlation coefficients, and the $\chi^{2}$ test was used for the categorical variables. The analyses were performed using PASW 18 (SPSS/IBM, Chicago, IL, USA) software and a two-tailed $p$-value less than 0.05 was considered to indicate statistical significance.

\section{Results}

\section{Demographic and clinical characteristics}

The average duration of psoriasis was 7-8 years. High sensitivity C-reactive protein (hsCRP) was higher in the psoriasis patient group than the control group (group 1: $1 \pm 0.8$; group 2: 0.6 $\pm 0.3, p<0.05$ ). Except for premature atrial extra beats no sustained atrial arrhythmia was detected in the Holter ECG. The demographic and clinical characteristics of the groups are shown in Table I. The average age of study participants in group 1 was 36 (20-60) and was 37 (20-54) in group 2; this difference was not significant $(p>0.05)$. Additionally, no statistically significant differences between the groups in terms of gender, BMI, smoking, waist circumference, systolic and diastolic blood pressure, fasting glucose, low-density lipoprotein ( $L D L)$ cholesterol, triglyceride and heart rate were revealed. The average NAPSI value was $23.9 \pm 5$, the PASI value was $3.4 \pm 1$, and the PSI value was $2.8 \pm 0.8$.

\section{Echocardiographic and electrocardiographic characteristics}

The echocardiographic and electrocardiographic characteristics of the groups are shown in Table II. No statistically significant differences were observed between the two groups in terms of LVEF, E wave, A wave, E/Em, DT, IVRT, LA diameter, LAVmax, LAVmin, LAVreservoir and LAGLSRa $(p>0.05)$. However, the LAGLS value was significantly lower in the patients in group 1 compared to control subjects in group 2 (group 1: $28.2 \pm 7.4$; group 2: 42.0 $\pm 3.7, p<0.05$ ). Additionally, the LAGLSRs value was significantly lower in the subjects in group 1 compared to group $2(p<0.05)$. The LAGLSRe value was also significantly lower in group $1 \mathrm{com}$ pared to group $2(p<0.05)$. With regard to the atrial conduction characteristics, TACT was significantly longer in patients in group 1 compared to the controls in group 2 (group 1: $103.5 \pm 3.7$; group 2: $99.1 \pm 4.4, p<0.05$ ). Additionally, PWD was higher in group 1 compared to group 2 (group 1: $59.5 \pm 4$; group 2: $47.1 \pm 2.9, p<0.05$ ).

\section{Evaluation of correlations}

A significant and negative moderate correlation was observed between LAGLS and TACT ( $r=$ $-0.57, p<0.05)$, and a significant negative strong correlation was also determined between LAGLS and disease duration $(r=-0.62, p<0.05)$. Additionally, a significant negative correlation was detected between LAGLS and PASI, NAPSI ( $r=-0.45$, $r=-0.60 ; p<0.05$ ) (Figure 3), while a significant negative moderate correlation was observed between LAGLS and PWD $(r=-0.65, p<0.05)$. A significant positive moderate correlation between TACT and PWD $(r=0.42, p<0.05)$ was determined. We also determined that a significant positive moderate correlation existed between TACT and disease duration $(r=0.52, p<0.05)$. 
Table I. Demographic and clinical characteristics of the groups

\begin{tabular}{|lccc|}
\hline Parameter & Group $1(n=40)$ & Group 2 $(n=40)$ & $P$-value \\
\hline Age [years] & $36(20-60)$ & $37(20-54)$ & $>0.05$ \\
\hline Sex (female/male) & $23 / 17$ & $21 / 19$ & $>0.05$ \\
\hline Body mass index [kg/m² & $24.7 \pm 7.7$ & $23.3 \pm 4.4$ & $>0.05$ \\
\hline Smoking, $n$ & 21 & 22 & $>0.05$ \\
\hline Waist circumference $[\mathrm{cm}]$ & $95(80-140)$ & $90.5(70-140)$ & $>0.05$ \\
\hline Systolic blood pressure $[\mathrm{mm} \mathrm{Hg}]$ & $120.4 \pm 20.2$ & $122.3 \pm 19.7$ & $>0.05$ \\
\hline Diastolic blood pressure $[\mathrm{mm} \mathrm{Hg]}$ & $75.8 \pm 11.4$ & $79.2 \pm 11.9$ & $>0.05$ \\
\hline Heart rate [beats/min] & $85.5(60-110)$ & $80(60-110)$ & $>0.05$ \\
\hline Fasting glucose $[\mathrm{mg} / \mathrm{dl}]$ & $90.6 \pm 13.6$ & $88.8 \pm 12.1$ & $>0.05$ \\
\hline LDL cholesterol [mg/dl] & $132.9 \pm 27.1$ & $123.7 \pm 32.1$ & $>0.05$ \\
\hline Triglycerides [mg/dl] & $147.8 \pm 27.1$ & $159.4 \pm 19.6$ & - \\
\hline Psoriasis Severity Index & $2.8 \pm 0.8$ & 0 & - \\
\hline Psoriasis Area and Severity Index & $3.4 \pm 1$ & 0 & - \\
\hline Nail Psoriasis Severity Index & $23.9 \pm 5$ & 0 & \\
\hline
\end{tabular}

Group 1 - psoriasis patients, group 2 - control group, $L D L$ - low-density lipoprotein; $p<0.05$ - a significant difference.

Table II. Echocardiographic and electrocardiographic characteristics of the groups

\begin{tabular}{|lccc|}
\hline Parameter & Group $1(n=40)$ & Group $2(n=40)$ & $P$-value \\
\hline LVEF $(\%)$ & $63 \pm 4.0$ & $64.6 \pm 3.3$ & $>0.05$ \\
\hline E wave $[\mathrm{m} / \mathrm{s}]$ & $0.68 \pm 0.1$ & $0.74 \pm 0.1$ & $>0.05$ \\
\hline A wave $[\mathrm{m} / \mathrm{s}]$ & $0.73 \pm 0.1$ & $0.74 \pm 0.06$ & $>0.05$ \\
\hline E/Em & $8.2(6-9)$ & $8(6-9)$ & $>0.05$ \\
\hline DT [ms] & $181.0 \pm 14.0$ & $178.0 \pm 7.0$ & $>0.05$ \\
\hline IVRT [ms] & $78.4 \pm 6.4$ & $81.5 \pm 7.2$ & $>0.05$ \\
\hline LA diameter [mm] & $35.8 \pm 2.2$ & $34.9 \pm 2.5$ & $>0.05$ \\
\hline LAV max. [ml] & $41.2 \pm 3.8$ & $39.3 \pm 3.3$ & $>0.05$ \\
\hline LAV min. [ml] & $13.1 \pm 2.8$ & $12.1 \pm 3.1$ & $>0.05$ \\
\hline LAV reservoir [ml] & $28 \pm 3.1$ & $28.1 \pm 3$ & $<0.05$ \\
\hline LAGLSs $(\%)$ & $28.2 \pm 7.4$ & $42.0 \pm 3.7$ & $<0.05$ \\
\hline LAGLSRs [s $\left.{ }^{-1}\right]$ & $1.9 \pm 0.5$ & $2.2 \pm 0.3$ & $<0.05$ \\
\hline LAGLSRe [s $\left.{ }^{-1}\right]$ & $1.3(1-1.9)$ & $1.5(1.1-1.9)$ & $>0.05$ \\
\hline LAGLSRa [s $\left.{ }^{-1}\right]$ & $1.4 \pm 0.1$ & $1.4 \pm 0.2$ & $<0.05$ \\
\hline TACT [ms] & $103.5 \pm 3.7$ & $99.1 \pm 4.4$ & $<7.1 \pm 2.9$ \\
\hline PWD [ms] & $59.5 \pm 4$ & 0.05 \\
\hline
\end{tabular}

Group 1 - psoriasis patients, group 2 - control group, LVEF - left ventricular ejection fraction, DT - deceleration time, IVRT - isovolumetric relaxation time, LA - left atrium, LAV max - LA maximum volume, LAV min - LA minimum volume, LAV reservoir - LAVmax. - LAVmin, LAGLSs - global peak systolic LA myocardium strain during left ventricular systole, LAGLSRs - global peak systolic positive LA myocardial strain rate during left ventricular systole, LAGLSRe - global peak negative LA myocardial strain rate during early ventricular diastole, LAGLSRa-global peak negative LA myocardial strain rate, TACT - total atrial conduction time, PWD-P-wave duration difference; $p<0.05$ indicates a significant difference. 


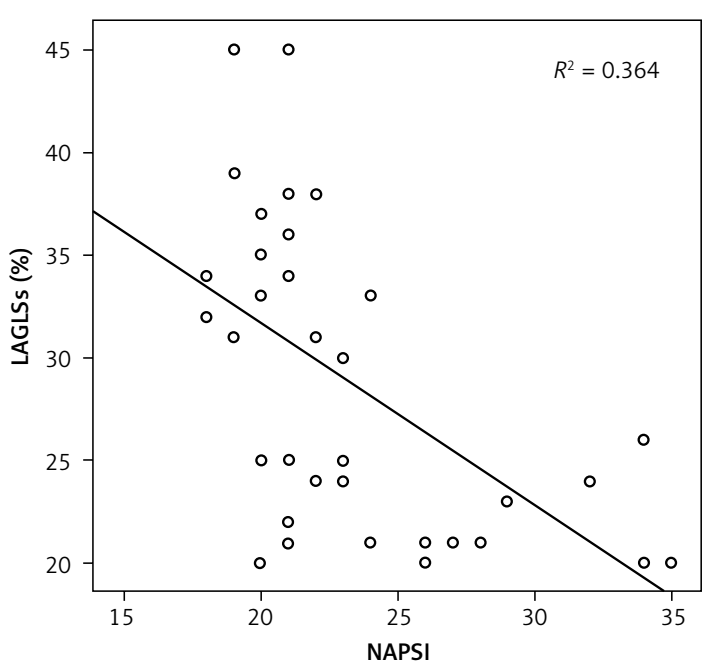

Figure 3. Correlation of left atrial systolic global longitudinal strain (LAGLSs) with the Nail Psoriasis Severity Index (NAPSI)

\section{Discussion}

In the current study, we determined that LAGLS decreased, TACT was prolonged, and PWD was increased in psoriasis patients. Additionally, a strong negative relation between LAGLS and TACT was found. While a negative moderate correlation between LAGLS and PWD was observed, there was a positive moderate correlation between TACT and PWD. We detected a strong negative correlation between LAGLS and disease duration and parameters indicating severity of disease, while a positive moderate correlation was observed between TACT and disease duration and parameters indicating severity of disease. The incidence of psoriasis is between $0.6 \%$ and $6.5 \%$ in Europe [15]. It has been reported that psoriasis patients exhibit increased cardiovascular morbidity and mortality due to mechanisms such as chronic inflammation, endothelial dysfunction, and oxidative stress [16]. Therefore, it is important to predict the potential arrhythmias that cause morbidity and mortality in this patient group, including atrial arrhythmia. The increase in PWD observed in the psoriasis patient group suggests that chronic inflammation plays a role in the development of atrial conduction disorders. The AF is the most common atrial arrhythmia, and the increase in PWD, the prolongation of TACT, and the decrease in LAGLS are essential factors that facilitate the prediction of this arrhythmia. Ahlehoff et al. [4] demonstrated that the risk of AF increased in patients with psoriasis. Atrial fibrillation is the most common arrhythmia and confers a substantial stroke risk, ranging from $<1 \%$ to $>20 \%$ in the absence of anticoagulation [17]. The $\mathrm{AF}$ also may lead to heart failure, pulmonary embolism, and acute coronary syndrome. Therefore, the ability to predict AF is vital to prevent morbidity and mortality. As shown in our study, the increase of PWD and the prolongation of TACT, which indicated the presence of a disorder in sinus impulse conduction, are important in terms of predicting AF. Additionally, the decrease in LAGLS, which indicated the deformation of the atrial myocardium and the subclinical dysfunction, will facilitate the early diagnosis of atrial conduction disorders. The LAGLS and TACT may be utilised as new echocardiographic markers in the early diagnosis of subclinical electrophysiological changes in patients with psoriasis. Tsai et al. found, in a study performed on 52 patients with paroxysmal AF [18], that there was a strong relationship between AF and LAGLS. Various other studies have also shown that LAGLS decreased in response to physiopathological diseases related to the left atrium such as atrial fibrillation, heart failure, valvular diseases, hypertension, and cardiomyopathy [19-22]. While a decrease in LAGLS value was also observed in the current study, there was a strong negative relationship between the decrease and the disease duration. This result may stem from the fact that chronic inflammation and oxidative stress affect the left atrium myocardium. As the disease duration increases, the inflammation may lead to the development of fibrosis on the atrial wall. This also prolongs TACT by blocking the atrial conduction pathways with fibrosis. Atrial conduction abnormalities and left atrial mechanical dysfunction were demonstrated in the psoriasis patient group in the current study and a positive strong correlation with disease duration and severity was observed $[23,24]$. This result is important for the ability to predict AF and other atrial arrhythmias. The capability to determine electrophysiological changes at the subclinical level will contribute to a reduction in cardiovascular morbidity and mortality.

\section{Clinical applicability of the study results}

The results of the current study will facilitate identification of patients who have an increased risk of developing cardiovascular disorders. Additionally, early detection of potential risk factors can facilitate the implementation of lifestyle modifications, including changes to the diet, exercise, and smoking cessation in patients exhibiting an increased cardiovascular risk. Intense medical treatments aimed at decreasing inflammation and oxidative stress would be beneficial for patients with advanced disease duration and severity, and would likely decrease cardiovascular morbidity and mortality. ECG, speckle tracking echocardiography, and atrial tissue Doppler imaging may be utilised as screening tests to assess the cardiovascular risk in patients undergoing systemic treatment. The establishment of preventive treatment strategies early during disease progression will be 
especially useful in young patients who exhibit a moderate or severe level of the disease.

The main limitations of the current report were the small number of patients included in the study, and the fact that no long-term follow-up of the patients was performed, especially those addressing atrial arrhythmia. Since our study was cross-sectional in design, our results cannot demonstrate causality.

In conclusion, we determined in the current study that LAGLS decreased, TACT was prolonged, and PWD increased in patients with psoriasis. There is a relationship between TACT, LAGLS, PWD and the duration and severity of disease. These results demonstrate that $L A G L S$, TACT, and PWD are important markers with the ability to predict $\mathrm{AF}$, and are capable of detecting the subclinical electrophysiological changes in psoriasis patients. Since anti-arrhythmic preventive measures may not be effective in this specific patient subset, we believe that clinicians should be alert for patients experiencing dyspnoea and palpitations, knowing the increased prevalence of AF.

\section{Conflict of interest}

The authors declare no conflict of interest.

\section{References}

1. Christophers E, Mrowietz U. Psoriasis. In: Dermatology in General Medicine. $6^{\text {th }}$ edn. Fitzpatrick TB, et al. (eds.). McGraw-Hill, New York 2003; 407-25.

2. Braun-Falco O, Plewig G, Wolff HH, et al. Dermatology. $2^{\text {nd }}$ edn. Springer-Verlag, Berlin 2000; 585-607.

3. Bonifati C, Ameglio F. Cytokines in psoriasis. Int J Dermatol 1999; 38: 241-51.

4. Ahlehoff $\mathrm{O}$, Gislason GH, Jørgensen $\mathrm{CH}$, et al. Psoriasis and risk of atrial fibrillation and ischaemic stroke: a Danish Nationwide Cohort Study. Eur Heart J 2012; 33: 2054-64.

5. Biyik I, Narin A, Bozok MA, et al. Echocardiographic and clinical abnormalities in patients with psoriasis. J Int Med Res 2006; 34: 632-9.

6. Tsuda S, Maeyama Y, Yamamoto N, et al. Secondary amyloidosis complicating arthropathic psoriasis. Clin Exp Dermatol 1996; 21: 141-4.

7. Centurion OA. Clinical implications of the P wave duration and dispersion: relationship between atrial conduction defects and abnormally prolonged and fractionated atrial endocardial electrograms. Int J Cardiol 2009; 134: 6-8.

8. Weijs B, de Vos CB, Tieleman RG, et al. Clinical and echocardiographic correlates of intra-atrial conduction delay. Europace 2011; 13: 1681-7.

9. Lang RM, Bierig M, Devereux RB, et al. Recommendations for chamber quantification: a report from the American Society of Echocardiography's Guidelines and Standards Committee and the Chamber Quantification Writing Group, developed in conjunction with the European Association of Echocardiography, a branch of the European Society of Cardiology. J Am Soc Echocardiogr 2005; 18: 1440-63.
10. Merckx KL, De Vos CB, Palmans A, et al. Atrial activation time determined by transthoracic Doppler tissue imaging can be used as an estimate of the total duration of atrial electrical activation. J Am Soc Echocardiogr 2005; 18: 940-4.

11. Louden BA, Pearce DJ, Lang W, et al. Psoriasis Area Severity Index (SPASI) for rating psoriasis severity in clinic patients. Dermatol Online J 2004; 10: 7.

12. Amornpinyokeit N, Asawanonda P. 8-Methoxypsoralen cream plus targeted narrowband ultraviolet B for psoriasis. Photodermatol Photoimmunol Photomed 2006; 22: 285-9.

13. Rich P, Scher RK. Nail Psoriasis Severity Index: a useful tool for evaluation of nail psoriasis. J Am Acad Dermatol 2003; 49: 206-12.

14. Michelucci A, Bagliani G, Colella A, et al. P wave assessment: state of the art update. Card Electrophysiol Rev 2002; 6: 215-20.

15. Chandran V, Raychaudhuri SP. Geoepidemiology and environmental factors of psoriasis and psoriatic arthritis. J Autoimmun 2010; 34: 314-21.

16. Flammer AJ, Ruschitzka F. Psoriasis and atherosclerosis: two plaques, one syndrome? Eur Heart J 2012; 33: 1989-91.

17. Fauchier L, Philippart R, Clementy N, et al. How to define valvular atrial fibrillation? Arch Cardiovasc Dis 2015; 108: 530-39.

18. Tsai WC, CH Lee, CC Lin, et al. Association of left atrial strain and strain rate assessed by speckle tracking echocardiography with paroxysmal atrial fibrillation. Echocardiography 2009; 26: 1188-94.

19. Sirbu C, Herbots L, D'hooge J, et al. Feasibility of strain and strain rate imaging for the assessment of regional left atrial deformation: a study in normal subjects. Eur J Echocardiogr 2006; 7: 199-208.

20. Inaba Y, Yuda S, Kobayashi N, et al. Strain rate imaging for noninvasive functional quantification of the left atrium: comparative studies in controls and patients with atrial fibrillation. J Am Soc Echocardiogr 2005; 18: 729-36.

21. Cianciulli TF, Saccheri MC, Lax JA, et al. Two-dimesnional speckle tracking echocardiography for the assessment of atrial function. World J Cardiol 2010; 2: 163-70.

22. Saha SK, Anderson PL, Caracciolo G, et al. Global left atrial strain correlates with CHADS2 risk score in patients with atrial fibrillation. J Am Soc Echocardiogr 2011; 24: 506-12

23. Tasal A, Guvenc TS, Kul S, et al. Atrial conduction abnormalities in patients with psoriasis vulgaris. Kardiol Pol 2015; 73: 637-43.

24. Aksan G, Nar G, Soylu K, et al. Assessment of atrial electromechanical delay and left atrial mechanical functions in patients with psoriasis vulgaris. Echocardiography 2015; 32: 615-22. 